\title{
Software for CCTV systems design
}

\author{
Milan Adamek ${ }^{1, a}$ Michaela Barinova and Tomas Havir ${ }^{1}$ \\ 1 Tomas Bata University in Zlin, Faculty of Applied Informatics, Nam. T. G. Masaryka 5555, 760 05 Zlin, Czech Republic
}

\begin{abstract}
The article is focused on the software, which is used in the design of the CCTV systems. It shows tools available online, tools for PC and mobile applications. It describes the basic components of the camera systems, their characteristics and current trends in CCTV systems. Moreover, it compares two selected software tools, their features and supported functions. In the practical part, these tools are used for the design of a CCTV system and the whole process is described in detail.
\end{abstract}

\section{Introduction}

CCTV systems help to protect the property, can provide evidence, inform about traffic situations, indicate suspicious behavior of people, etc. It is obvious that the application of CCTV systems will gradually expand in the future hand in hand with the development of new technologies and a gradual decrease in production costs.

Designing a CCTV system involves a number of calculations and specifications that are subsequently used for the selection of suitable components with desired properties. These basic parameters include the CCTV lens focal length, depth of field, CCTV viewing angle, parameters for identification of persons, data bandwidth capacity, voltage drop, the backup HDD storage space for various formats and also the minimum required upload of the internet connection to stream all the cameras in the designed CCTV system. The calculations do not have to be done manually, since there is a wide range of software tools that perform these calculations. Today's software applications, in addition to the calculation of the CCTV system component parameters, also allow modeling of selected objects.

There are online tools currently available that are usually open to the public at the websites of CCTV component manufacturers. And of course there are also sophisticated software tools that must be installed prior to their use on the computer.

The paper focuses on the analysis of online tools that can be used for the determination of parameters of individual CCTV components. Detailed comparison is provided of sophisticated software tools that can be used to create 3D models of an object, including their internal equipment. The CCTVs of required parameters are then placed to the models.

\section{Online tools for CCTV systems design}

\subsection{Calculator of focal length}

Through the calculator it is possible to calculate the appropriate focal length; to maintain the required image quality, the following input parameters must be specified: resolution (in pixels per meter), the height and width of the scene and its distance from the lens. The function of this tool is not only one-way to calculate the focal length, but quite the contrary; after entering the focal length it is possible to calculate any of the above parameters, Fig. 1.

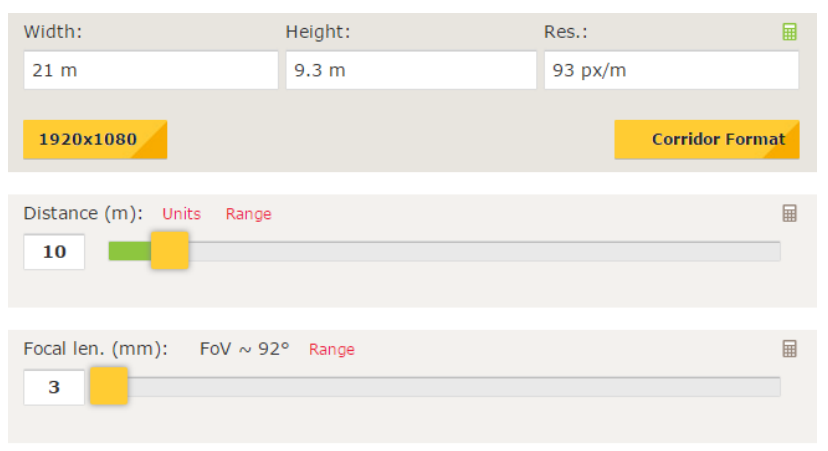

Figure 1. AXIS Lens calculator [1]

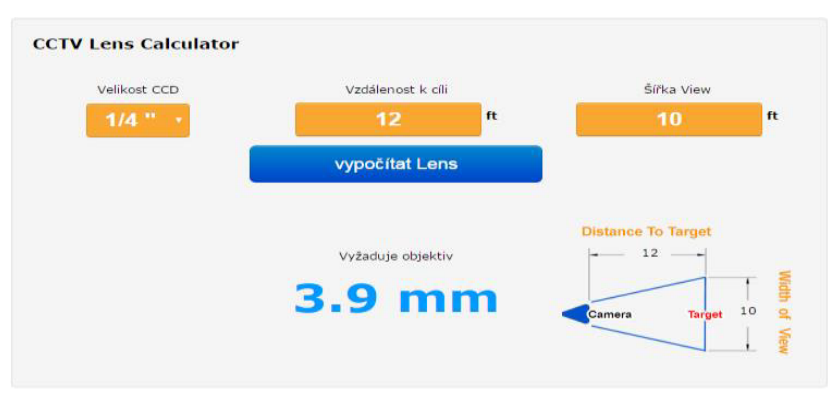

Figure 2. CCTV Lens calculator [2]

\footnotetext{
${ }^{\mathrm{a}}$ Corresponding author: adamek@fai.utb.cz
} 


\subsection{CCTV Designer}

Through this tool, you can design a CCTV system. It allows adding cameras, recording equipment, accessories or network elements to your own design; these elements can be selected from a list of components. A useful feature is specification of parameters of a new item. Designer lets you see a preview of the camera view; by a movement you can set the viewing angle, the height and distance of the monitored person. Depending on the CCTV and its parameters, a real shot of the person is displayed in the right side of the screen, which shows the image quality. You can also switch to a 3D mode, where the shot is displayed in 3D space. It is possible to move the graphical representation of the person's silhouette and check whether the image is of sufficient quality.

It is also possible to select the type of the scene; from the default scenes you can choose a busy street, parking place, office space, etc. Based on the choice made, the approximate size of one image is calculated. Depending on the CCTV number, type and time required for archiving, the needed storage capacity is calculated and a suitable recording device is selected accordingly.

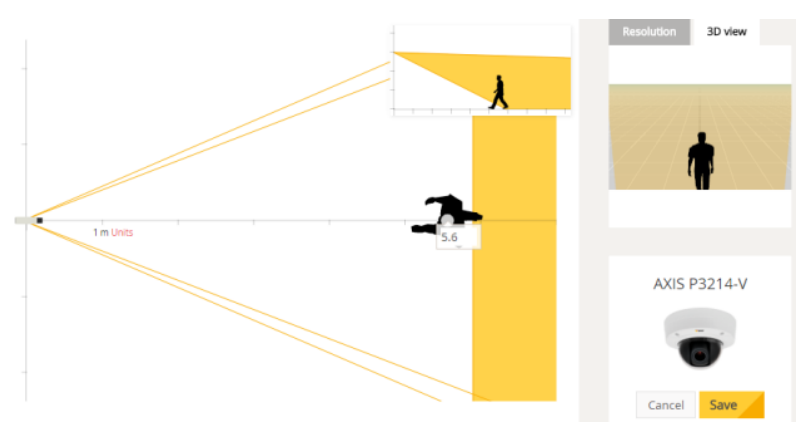

Figure 3. Design system [3]

\subsection{HDDs Calculator}

After entering the parameters of the selected CCTV system, the Calculator calculates the storage capacity of a hard drive in order to enter and save the time required for recording. This requires the following parameters: CCTV resolution, image frequency, number of CCTVs and required recording time. After entering of all said parameters, the Calculator calculates the required storage capacity.

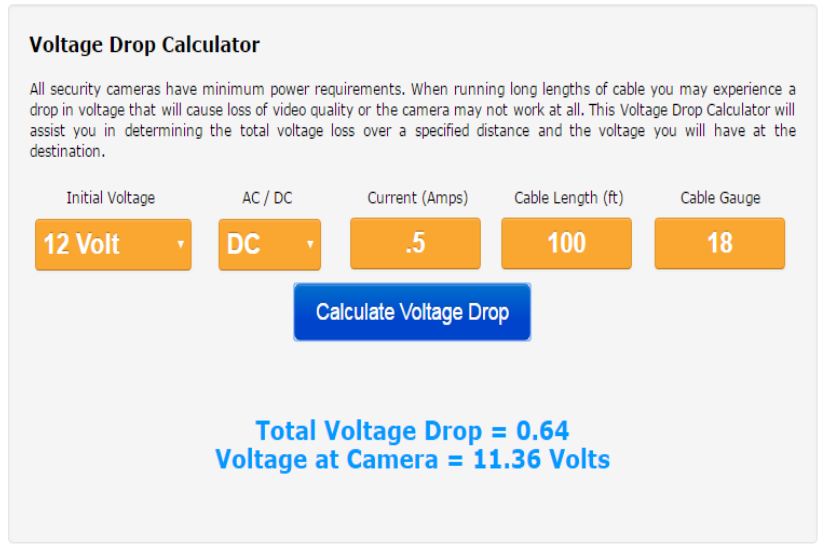

Figure 4. Voltage drop calculator

\subsection{Voltage Drop Calculator}

Calculator that can calculate the voltage drop across the transmission route. It is required to specify the values of voltage, current, cable length and cable gauge [4].

\subsection{Calculator with a support for Google Maps}

A tool that can display a security camera view on a map and its approximate image quality. It utilizes information from Google Maps and calculations which are performed on the basis of the specified parameters.

The camera can be placed anywhere in the world; if images captured for the Street View are available, a rough view of the scene will be showed on the right side of the screen. The tool also includes the function of automatic calculation of the pixel density and in the right side it shows a snapshot figure, which implies the quality of the image. The tool also allows changing the area that is monitored by the camera, and it accordingly recalculates the lens parameters and changes the image quality.

The CCTV properties can be defined manually and the parameters can be modified for various purposes. In the event that the software tool user decides on a particular CCTV model with predefined parameter settings, there is a choice of more than 2500 camera models from wellknown manufacturers.

Figure 5 shows a camera capturing a scene between two streets Masarykova and Nádražní in Brno. The upper part of the figure shows the parameters that can be changed as needed. In the left you can see the camera image and in the right an approximation of the real picture. In the lower right corner you can see the image quality of a person captured on camera; the person is showed as a drawn human silhouette in the left within the green triangle. [5]

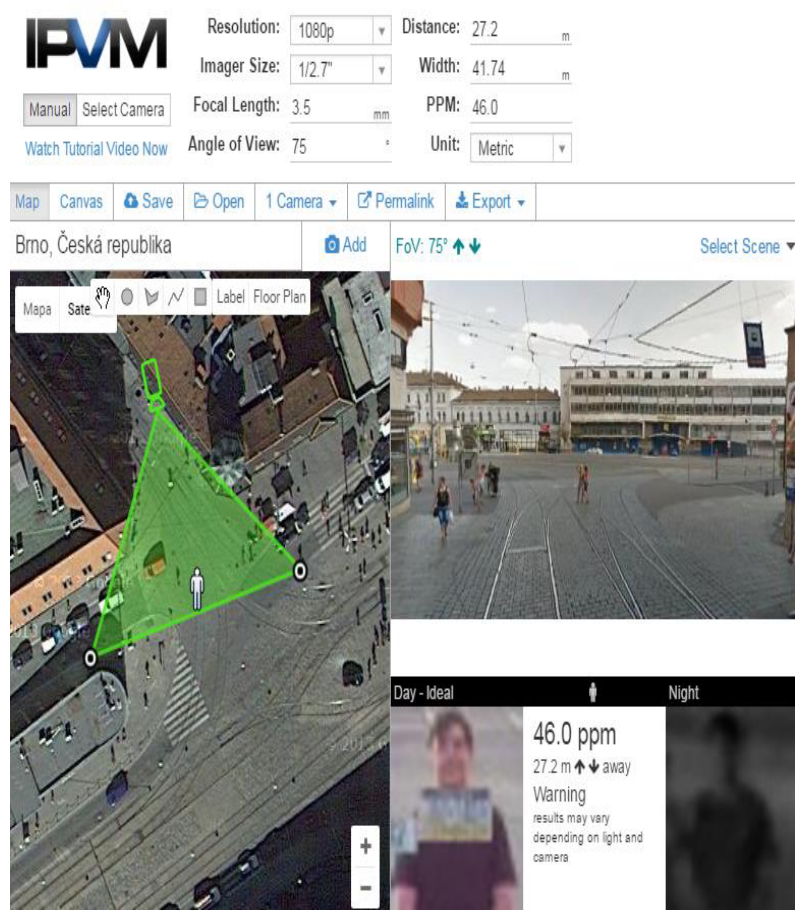

Figure 5. IPVM Calculator 


\section{Software for the PC}

\subsection{Microsoft Visio}

A simple 2D tool, which can create CCTV designs. The program includes a number of predefined blocks, which are standardized. Of course, the user can generate their own blocks with defined parameters via a library. The blocks are connected by lines using the connecting points.

Data from individual blocks can be interconnected with other Microsoft programs, such as Microsoft Excel or Microsoft SQL.

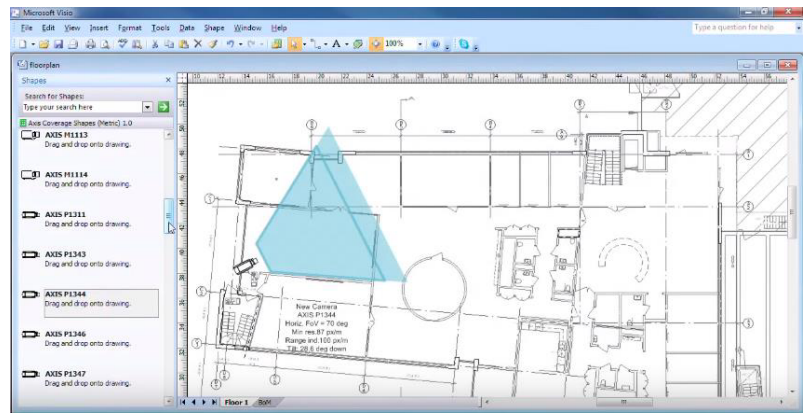

Figure 6. Example of CCTVs installation in Microsoft Visio [6]

\subsection{SketchUP}

Program for modeling in 3D space, which is not branch-specific, and therefore allows modeling of absolutely anything from individual interior elements for an entire house, or for example technical equipment or things that we use on daily basis.

Drawing is easier than in other professional design softwares such as CAD. Most models are created by drawing with classic tools such as pencil, ellipse or rectangle, and subsequently using the drag function allows creation of a 3D object. The sketchup contains a large library, in which you can find a lot of pre-defined objects created by users themselves. For example furniture, trees, equipment or individual instruments.

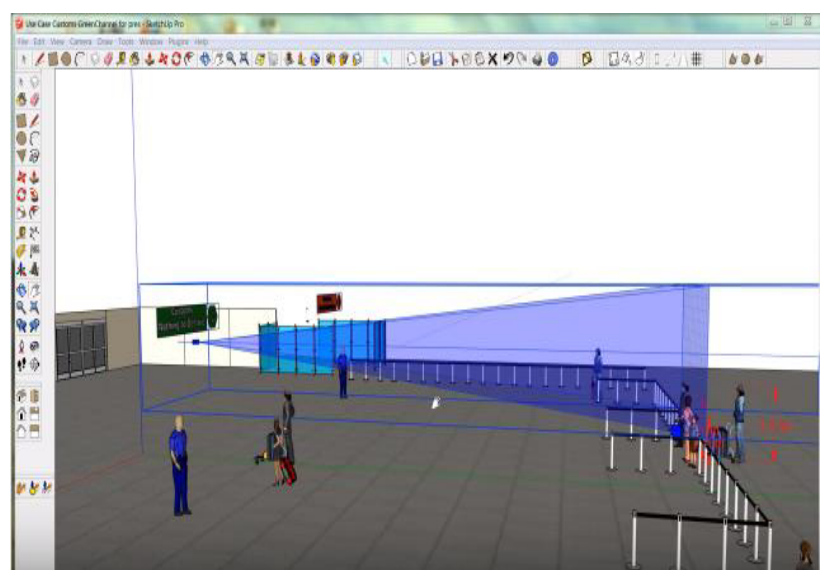

Figure 7. Example of SketchUP environment [7]

The feature that allows cooperation of the program with Google Earth enables the users to upload individual objects also from this application. For example, you can use the terrain, bumps or individual buildings. It is also possible to draw by means of importing photos. The resulting models can be exported into many file types. The dwg file types are also possible to use in CAD systems.

In CCTV systems, it is possible to insert individual cameras into 3D designs and monitor their properties. The camera can be modeled as a new object or you can download pre-defined cameras from Axis if needed. The creators of the program CCTVCAD developed an add-on for SketchUp that allows inserting cameras into objects objects and arbitrarily modify their properties. The result can be seen as a camera footage in 3D mode [7].

\subsection{AutoCAD}

Professional software which supports 2D drawing and 3D modeling.

The 3D modeling in this software has not become widely popular in the field of CCTV systems, mainly due to its complexity. However, drawing in $2 \mathrm{D}$ is quite the opposite and is used very frequently. It is mainly used for plotting of cable locations and placing of CCTV system components to a house layout. In AutoCAD you can also use the function for distance measurement for qualified estimation of the required amount of cabling.

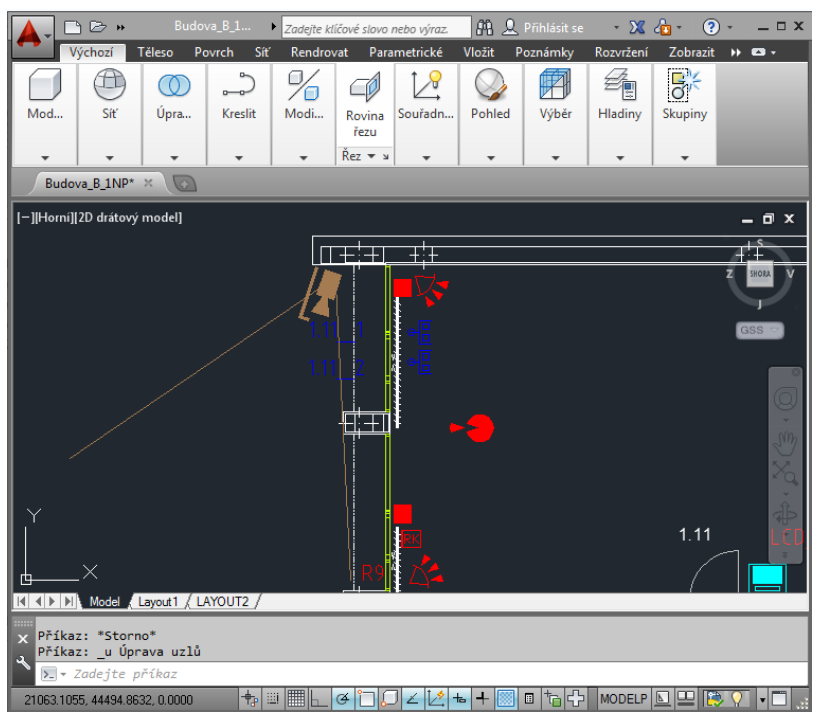

Figure 8. Ukázka půdorysu v prostředí AutoCAD - zakreslení slaboproudých komponent

\subsection{JVSG - CCTV design software}

Professional software, which facilitates the process of designing a CCTV system and makes an effort to eliminate the risk of potential errors or wrong configurations.

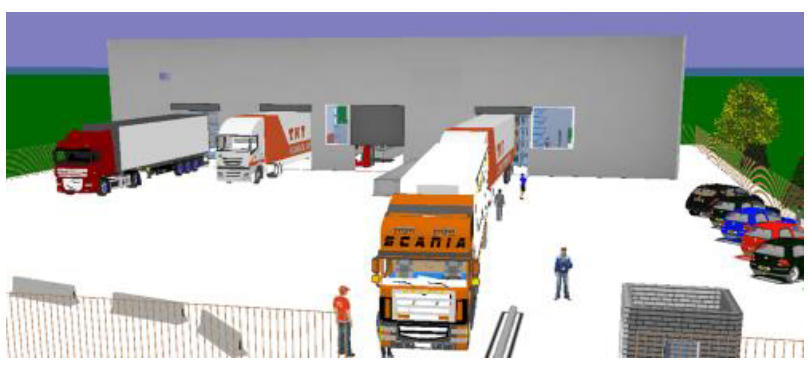

Figure 9. 3D objects in JVSG environment [7] 
Although the original title contains IP CCTV systems, the tool serves also for the design of analog systems.

A 3D model is created based on the plans of the object into which we place cameras with defined configurations and parameters. During the modeling it is possible to import external 3D objects in Collada formats.

When working in this environment after loading the object layout in JPEG, PNG, BMP, PDF, MS Visio or Google Earth formats you can be model walls, rooms, windows, doors and more. Subsequently, equipment and other objects are added into the object. This is followed by adding cameras, the number of which in one project can reach up to 150; this number allows proposal of extensive CCTV systems. The tool can calculate fields of vision, viewing angles and focal lengths. In the visual field it can calculate the image quality in relation to the distance of captured object. The vision fields can be divided into zones, the colors of which represents the video quality for detection.

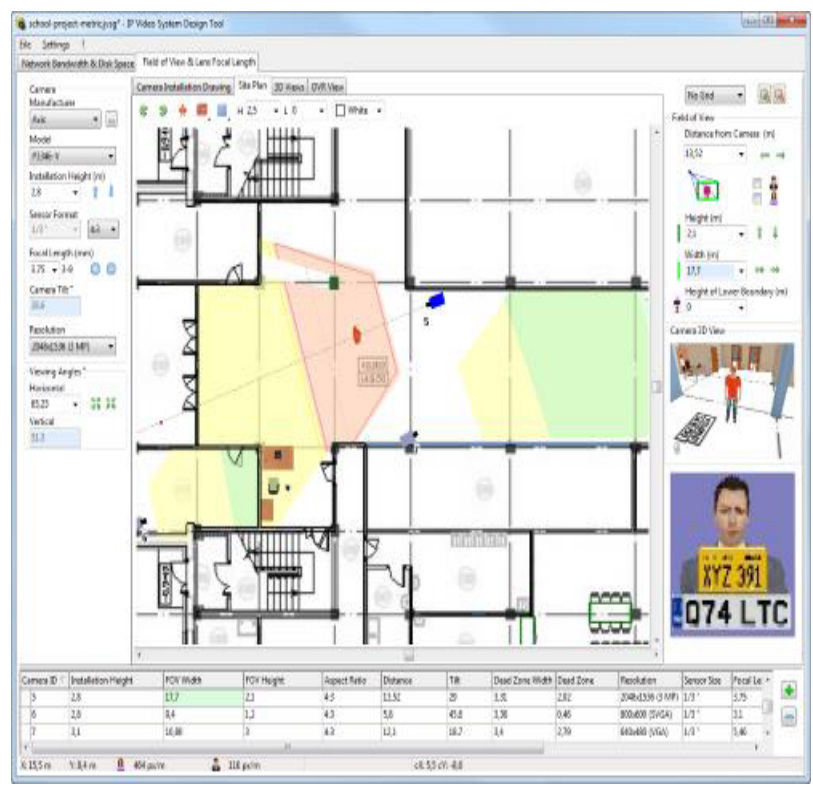

Figure 10. A layout with color zones in JVSG environment [7]

The software can display real views from all cameras in $3 \mathrm{D}$ mode and can also display the quality of a sample image in relation to the distance from the camera, all this in both day and night regimes. Another feature is displaying of the image quality when identifying the license plates of vehicles; this feature can be used, for example, when installing CCTVs at gates or entrances to buildings.

\subsection{CCTVCAD}

Comprehensive software for CCTV system designs which facilitates the calculations necessary for the selection of suitable system components. It can also calculate focal lengths, viewing angles and ranges of the cameras. Further it helps determine the parameters for the detection of persons, their identification or reading the license plates of vehicles.

Other features include calculating the depth of field, voltage drops and determination of the required light output for sufficient lighting of the scene.

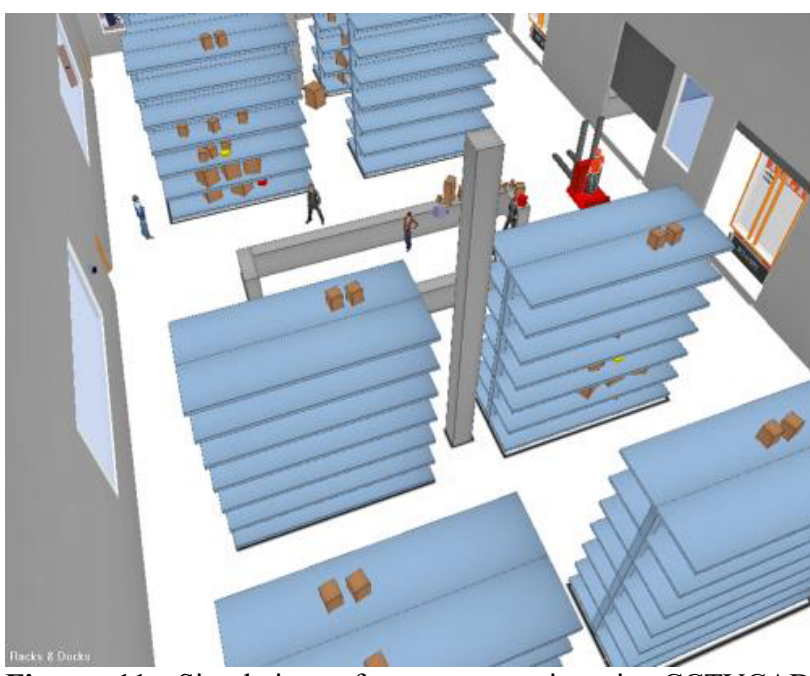

Figure 11. Simulation of a camera view in CCTVCAD environment [8]

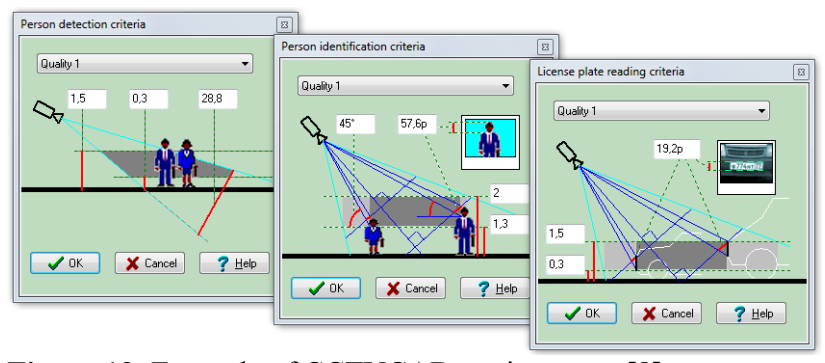

Figure 12. Example of CCTVCAD environment [8]

The entire facility can be drawn in $2 \mathrm{D}$ or $3 \mathrm{D}$ formats and the cameras can be placed in the designed model. They can be subsequently configured in order to see precise viewing angles, and realistic images in 3D. The images are displayed in a color corresponding to the image quality in relation to the distance from the camera. It is possible to set up our own color zones or choose from default options. The camera can be rotated in order to seek optimum angles to achieve the desired images. The software is also able of displaying zones of cameras that have lenses with high distortion.
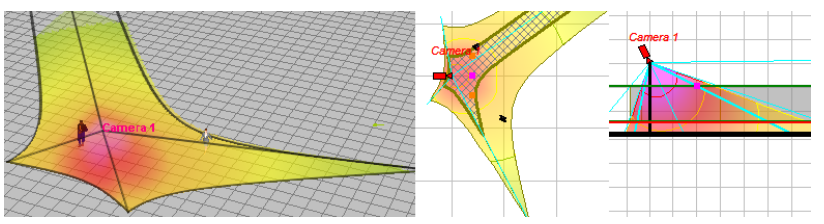

Figure 13. Simulation of a camera view in CCTVCAD environment [8]

Formats supported for the layout upload are BMP, JPG, EMF, WMF, PNG, GIF, TIF, PDF, DWG, DXF. Like the JVSG design tool, the CCTVCAD also contains its own 3D objects. Upload of external models into the layout is allowed from programs such as Autodesk 3Ds Max and SketchUp.

The software can simulate camera images based on camera settings and the parameters of the scene. For example, there are predefined scenes with artificial lighting, fog, reduced visibility, and others. Properties of light sources and camera sensitivities are also taken into consideration. You can also simulate infrared 
illumination of the cameras. According to the parameters, you can calculate the camera depth of field. Other displayed properties are image contrast, compression and rendering [8].

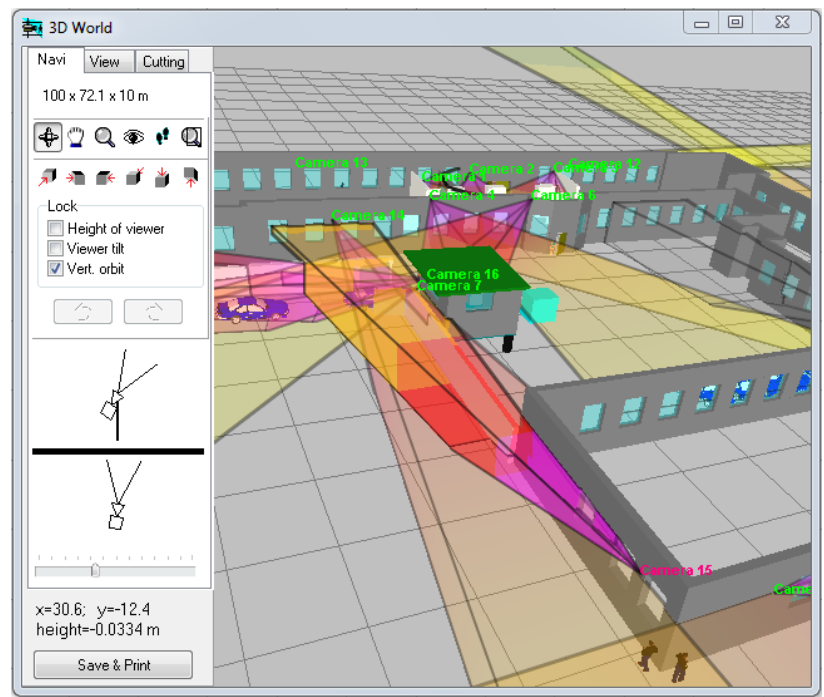

Figure 14. Scene parameters in CCTVCAD environment [8]

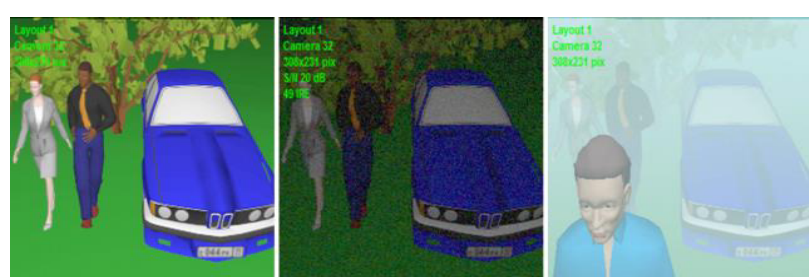

Figure 15. Scene parameters in CCTVCAD environment [8]

\section{CCTV design using JVSG}

An object layout created in AutoCAD was inserted as a background on which the object was modeled.

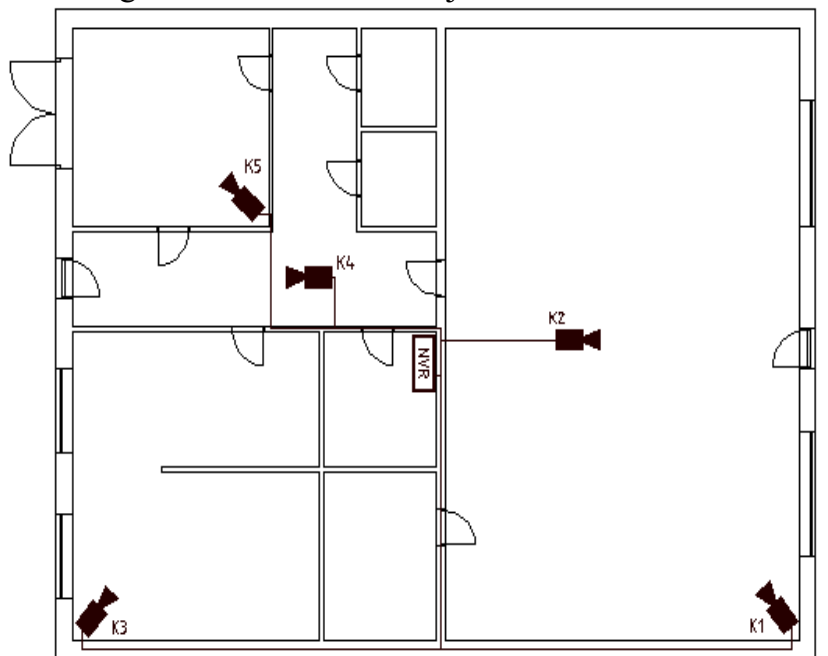

Figure 16. An object layout designed in AutoCAD environment

Individual offices in the building were equipped with office furniture using the sources from libraries in a Collada format. The building also includes storage rooms equipped with storage shelves and goods. Also the shelves were imported in the Collada format.

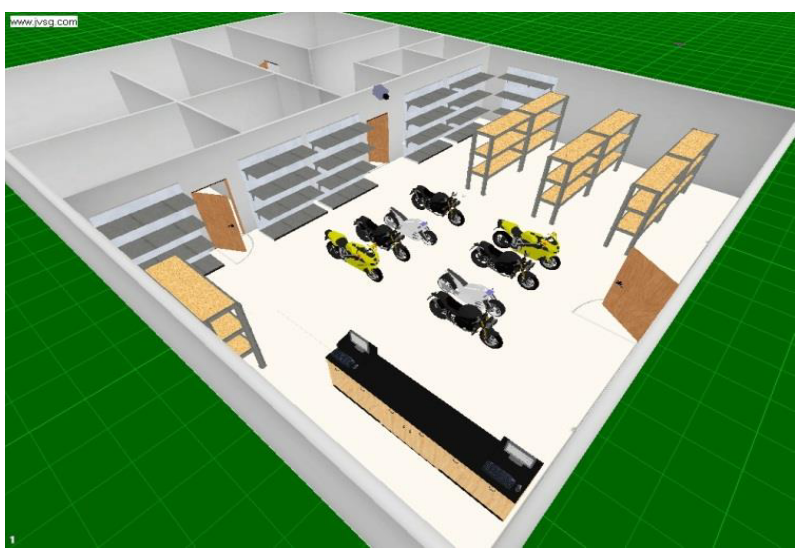

Figure 17. Model of a store premises designed in JVSG environment

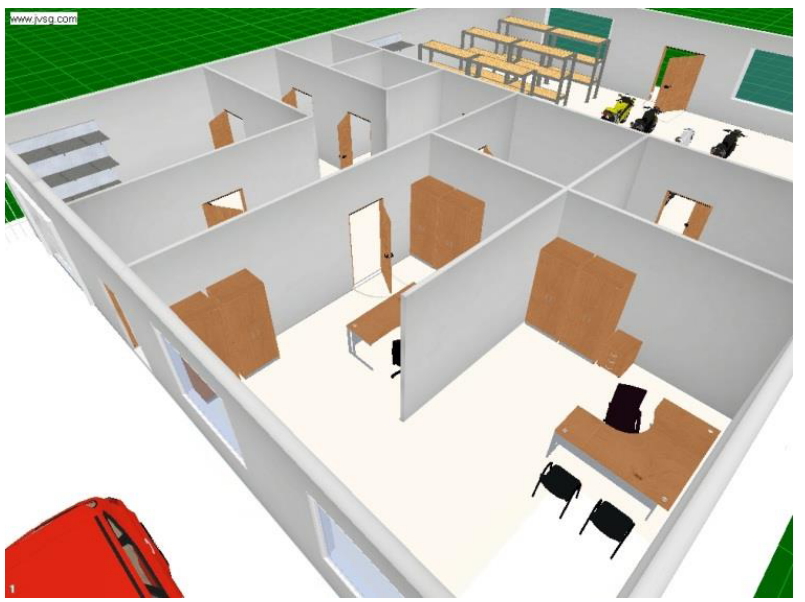

Figure 18. Model of office premises designed in JVSG environment

\subsection{Choosing the cameras and their location in JVSG studio}

The facility has been used Five IP cameras in a dome design were used in the building connected by twisted pair cabling to an NVR recording device in the utility room. Cameras 1 and 2 were located in the sale space. Camera 3 is located in an office with a safe with money placed in a closet. Camera 4 captures the corridor and camera 5 is placed in the service department.

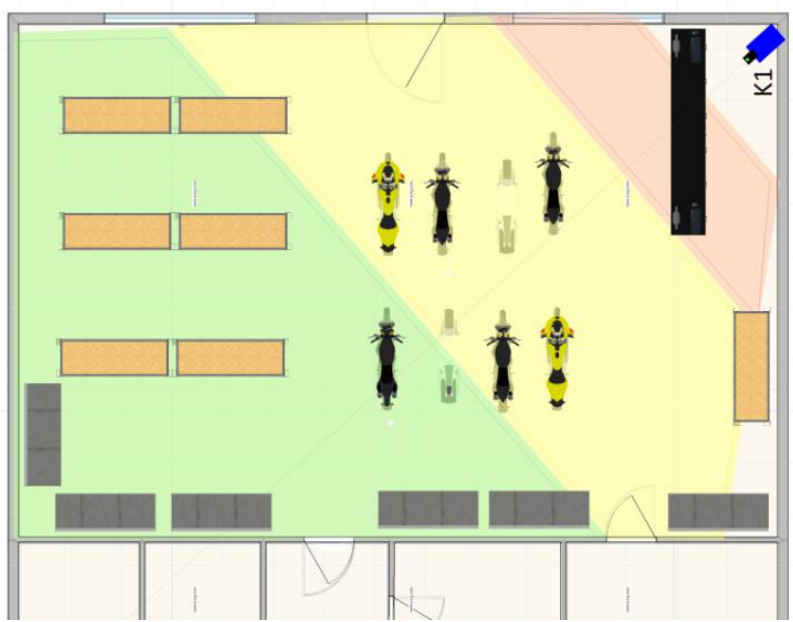

Figure 19. Location of camera $\mathrm{K} 1$ in a storage room of modeled object, created in JVSG environment 
The cameras of required properties (focal length) were chosen based on the simulation steps in JVSG environment and their locations within the model object were selected.

Figure 19 shows the location of camera K1 in the modeled object warehouse, Figure 20 represents the view of K1, which was created in JVSG Studio.

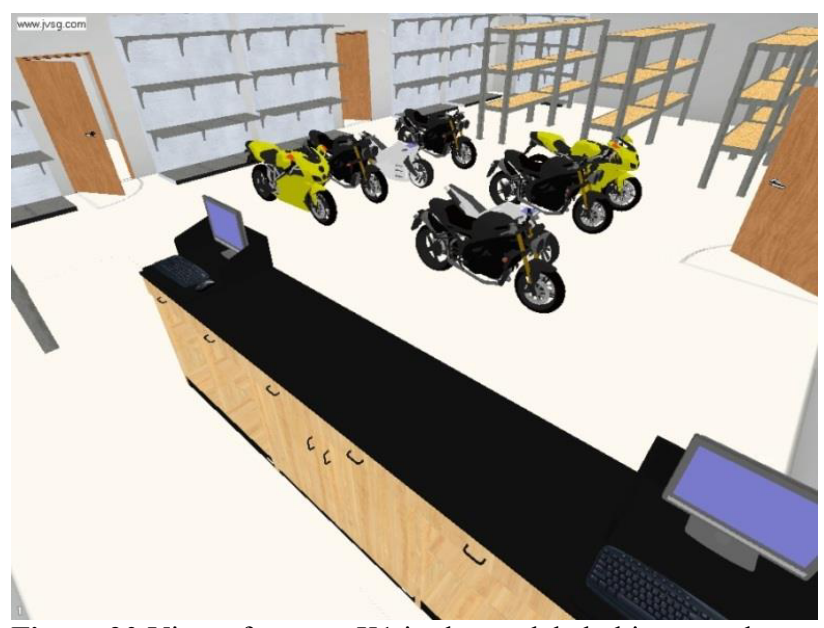

Figure 20 View of camera K1 in the modeled object warehouse designed in JVSG environment

The CCTV system includes five IP cameras that are connected via twisted-pair cabling to a NVR recording device. The NVR is located in the utility room and is equipped with a backup in case of power failure. A security and emergency alarm system (PZTS) centre is also present in the utility room, therefore this space is monitored by a PIR motion detector.

Cameras K1 to K5 represent IP cameras with varifocal lens of a $2.8-12 \mathrm{~mm}$ and image sensor of 1 / 2.8 "Sony. The cameras have a built-in IR illuminator, which reaches up to 30 meters, which is sufficient for the object distance. $12 \mathrm{~V} / 1 \mathrm{~A}$ is via PoE. The maximum camera resolution is $1080 \mathrm{p}$ and a compression of H.264 is set at a frequency of 15 images per second. The focal length settings of each camera is described in Table 1.

Table 1 Focal lengths of cameras K1 to K5

\begin{tabular}{|c|c|}
\hline Camera & Focal length $(\mathbf{m m})$ \\
\hline K1 & 2,9 \\
\hline K2 & 6,3 \\
\hline K3 & 2,9 \\
\hline K4 & 2,9 \\
\hline K5 & 3 \\
\hline
\end{tabular}

\subsection{NVR recording device}

For the CCTV system a network device was selected that can record simultaneously eight 2-megapixel cameras at a frequency of 15 images per second. All camera ports have PoE power connections, so it is not necessary to provide power for individual cameras. The NVR can be connected to Internet Network via a WAN port. The recording can be made on up to two 4TB drives. Supported are the Three types of compression are supported, namely the H.264, MPEG4 and MJPEG; the H.264 was chosen for the object [9].

\subsection{Hard drive}

The requirement for video record archiving is set up to the last four days. For installation, a hard drive with a capacity of 500 gigabytes was chosen, which is sufficient for that purpose. When a motion is detected within the camera field of vision, the cameras record at full quality; without motion detection they record at a lower resolution.

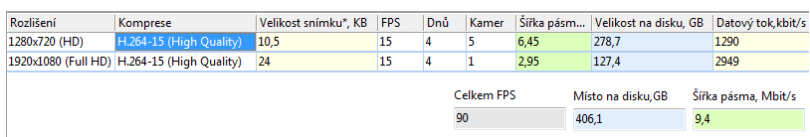

Figure 21 Calculation of a hard drive capacity for record within the last four days

\section{Conclusion}

Online software tools for designing CCTV systems are tools that are easier to use and in most cases solved by means of calculators. The tools can usually calculate the basic parameters of a CCTV system, which are often not sufficient for modeling of objects in which the CCTV system is to be located. Second type is represented by software tools which, once installed on a PC, are much more sophisticated environment with support of a wide range of libraries. They allow creating of $3 \mathrm{D}$ models of buildings and their interior equipment. Cameras with defined parameters are subsequently placed into the models. The tools can then simulate the images captured by the cameras and show previews of real footage. The last type of software are mobile applications. They predominantly operate on the same principle as the online tools.

\section{Acknowledgement}

This work was supported by the Ministry of Education, Youth and Sports of the Czech Republic within the National Sustainability Programme project No. LO1303 (MSMT-7778/2014) and also by the European Regional Development Fund under the project CEBIA-Tech No. CZ.1.05/2.1.00/03.0089.

\section{References}

1. Axis - Portál nástrojů [online]. 2016 [cit. 2016-0502]. In: http://www.axis.com/cz/cs/tools

2. Security Camera King - CCTV Calculators [online]. Boca Raton, 2016 [cit. 2016-05-02]. In: http://www.securitycameraking.com/calc.html

3. NAS Servery [online]. 2016 [cit. 2016-05-02]. In: http://www.nasservery.cz/ 
4. Security Camera King - CCTV Calculators [online]. Boca Raton, 2016 [cit. 2016-05-02]. In: http://www.securitycameraking.com/calc.html

5. Camera Calculator / Design Software [online]. Sanborn, 2016 [cit. 2016-05-02]. In: http://ipvm.com/calculator

6. SketchUP [online]. 2014 [cit. 2016-05-02]. In: http://www.cadtutorial.cz/sketchup/sketchup_uvod_d o_3d_modelovani.php

7. JVSG: CCTV Design Software [online]. 2016 [cit. 2016-05-02]. In: http://www.jvsg.com/

8. CCTVCAD Software [online]. Perm, Russia, 2016 [cit. 2016-05-02]. In: http://www.cctvcad.com/

9. STASANET - Bezpečnostní technologie [online]. Praha, 2016 [cit. 2016-05-02]. In: https://www.stasanet.cz/ 